\title{
Cost effectiveness analysis of cervical cancer screening in women until age 70
}

\author{
James C. Quon* \\ School of Public Health, University of Alberta, Edmonton, AB, Canada \\ Received: 15 August 2018 \\ Accepted: 06 September 2018 \\ *Correspondence: \\ Dr. James C. Quon, \\ E-mail: jcquon@ualberta.ca \\ Copyright: $\odot$ the author(s), publisher and licensee Medip Academy. This is an open-access article distributed under \\ the terms of the Creative Commons Attribution Non-Commercial License, which permits unrestricted non-commercial \\ use, distribution, and reproduction in any medium, provided the original work is properly cited.
}

\begin{abstract}
Background: 2017 US Preventive Services Task Force guidelines for screening cervical cancer and pre-malignant lesions advise that screenings cease for women over age 65 , with qualifications. Recent literature has identified significant discrepancies in rates of cervical cancer in older women - if hysterectomies in this patient population is accounted for, cervical cancer incidence does not decline with age as previously established. This adjusted incidence of cervical cancer necessitates a re-examination of current practice.

Methods: This study seeks to demonstrate the utility of extending the cervical cancer screening age recommendations to age 70 . Cost effectiveness will be estimated, from a payer perspective, of extending screening to age 70 for the United States women's population in those who have not undergone hysterectomy or otherwise been treated for past cervical cancer or premalignancy. A Markov model was constructed to project outcomes in a hypothetical cohort of 10000 women aged 65 to 70, with a time horizon of lifetime. A Probability Sensitivity Analysis determined the robustness of the result, and the Incremental Cost-effectiveness Ratio (ICER) is charted.

Results: The economic evaluation of screening compared to none in this population was determined to be cost effective, with an ICER demonstrating a cost benefit, and Quality Adjusted Life Year (QALY) benefit, to extended screening.

Conclusions: The sensitivity analysis confirms the robustness of this result. Implementing extended screening guidelines could potentially be a significant gain for both patients and society.
\end{abstract}

Keywords: Cancer screening, Cervical cancer, Economic analysis, Gynecology, Primary care, Prevention

\section{INTRODUCTION}

Current guidelines for screening of cervical cancer and pre-malignant lesions advise that screenings cease for the majority of women over the age of 65 -those who have not had detectable dysplasia in the previous ten years. ${ }^{1,2}$ In the United States, recent literature has identified significant discrepancies in rates of cervical cancer in older women-if hysterectomies in this patient population are properly accounted for, cervical cancer incidence does not decline with age as previously established. ${ }^{3}$ This adjusted incidence of cervical cancer necessitates a reexamination of current practice. Miscalculated incidence rates have left a significant segment of elderly women unscreened, and potentially untreated for cervical dysplasia, and cancer. Subsequently, the impact on healthcare costs, both in health and financially, of this situation has gone unexamined.

This study examines the cost effectiveness of increasing the current recommended age of screening for cervical cancer up until the age of 70- improved screening guidelines requires evaluation, through incorporation of more accurate epidemiologic data in economic models. The costs of screening every five years with a combination of Papanicoloau cytology test and HPV 
DNA test, with subsequent treatment, are considered against the costs of cervical cancer detected at the symptomatic stages at which elderly women would currently present in lieu of screening. Our study investigates the potential cost effectiveness and impact of expanded screening guidelines for the high-risk population of elderly women.

\section{METHODS}

\section{Model}

A Markov model (Figure 1) was constructed to project outcomes in a hypothetical cohort of 10000 women aged 65 to 70. The model is an appropriately outlined disease progression of cervical dysplasia to either treatment, or to cancer onwards to death due to metastatic disease.

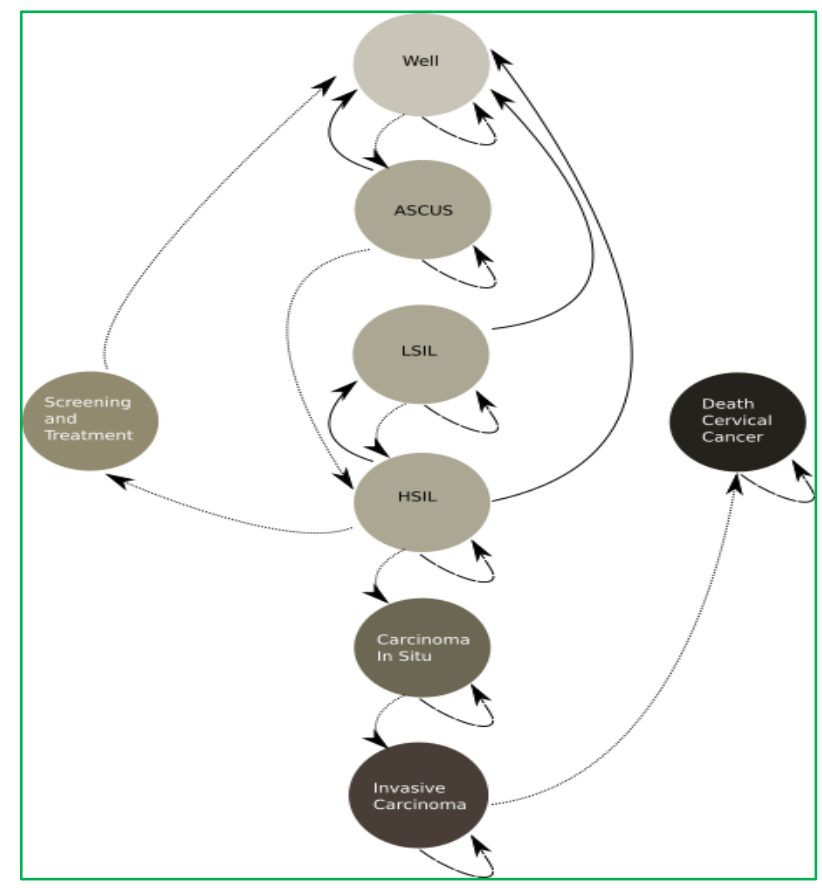

Figure 1. Markov model, cervical dysplasia, with progression to cancer.

At the outset, all members of the cohort will be healthy. Transition states will represent the clinical spectrum involved in cervical cancer diagnosis in regard to cytological and clinical stages as outlined by the Bethesda system, terminating with death from cervical cancer, or reversion to a healthy state via positive screening and treatment. ${ }^{4}$

Markov cycles will last 6 months, based upon mean regression times from disease states to a healthy state. ${ }^{5}$ Time horizon of the study will be lifetime - current life span is 81 for women in the US, subsequently there will be 32 cycles. Progression of disease states average significantly longer than six months. There will be an absorbing state of death.

\section{Event probabilities}

The Markov model makes necessary concessions to the availability of suitable data used to inform event probabilities. Transitions among pre-cancerous states is as comprehensive as possible-as LSIL and HSIL states are often treated upon detection, there are no directly observed progressions of disease in literature for the population we are examining. Probabilities in these instances were sourced from studies in which transitions were extrapolated from study data of LSIL and HSIL prevalence rates. $^{6}$

Assumed transition states probabilities have been calculated on the basis that each probability should sum to zero-so that the probability that a particular state remains in the next cycle, is the difference between 1 and the probabilities of progression and regression taken from the literature (following correction to the six-month cycle used in the model).

\section{Costs and preferences}

Costs will be considered from a societal perspective and measured in dollars per QALY using established EQ5D scores (Table 1). Costs were derived from the American Medical Association's 2015 CPT Coding guides and are intended to reflect the entirety of payer cost for each state in regard to the screening, and/or treatment cost for each state in the Markov model. A discount rate of $1.5 \%$ per six-month cycle will be used. Preference weights (Table 1) were taken from studies determining EQ5D scores in regard to specific stages of disease or health as described in the model's states. Refer to Table 1 for the probabilities, costs, and preferences utilized for the study.

\section{Economic analysis}

The Markov model, with aforementioned inputs, produced an Incremental Cost Effectiveness Ratio (ICER), which allows for a direct estimation in real world costs and impacts as to the utility of improved screening guidelines. Costs and preference weights (used to determine QALY's) were placed in the designed Markov model along with the simulated cohort in order to produce a final comparison between the costs and impacts of screening relative to no screening.

\section{Univariate sensitivity analysis}

Selected parameters from the Markov model's costs, preferences, and probabilities were varied in order to determine the effect of potentially plausible variation on the results of the model. Critical values were systematically altered and the resulting ICER values were placed in a tornado diagram to analyze the effect in relation to the base case ICER point estimate and compare the sensitivity of the model to an array of parameter variations. 


\section{Probabilistic sensitivity analysis}

In order to provide the likelihood that the ICER is within an acceptable range, considering the potential variation of all parameters at once, a cost effectiveness cloud is created.

Table 1: Base case probabilities, costs, and utility scores.

\begin{tabular}{|c|c|c|c|c|}
\hline Group & Parameter & Base Case & Distribution & Reference \\
\hline \multirow[t]{18}{*}{ No screening cohort } & Probability WELL progresses to ASCUS & 0.02070 & Uniform & $(\text { Edelman, et al })^{20}$ \\
\hline & Probability WELL stays & 0.96710 & Uniform & Assumption \\
\hline & Probability WELL progresses to LSIL & 0.01220 & Uniform & $(\text { Edelman, et al })^{20}$ \\
\hline & Probability ASCUS progresses to HSIL & 0.00450 & Uniform & $(\text { Walker, et al })^{18}$ \\
\hline & Probability ASCUS regresses to WELL & 0.17048 & Uniform & $(\text { Melnikow, et al) })^{21}$ \\
\hline & Probability ASCUS stays & 0.82503 & Uniform & Assumption \\
\hline & Probability LSIL progresses to HSIL & 0.02573 & Uniform & $(\text { Cantor, et al })^{6}$ \\
\hline & Probability LSIL regresses to WELL & 0.07400 & Uniform & $(\text { Cantor, et al })^{6}$ \\
\hline & Probability LSIL stays & 0.90027 & Uniform & Assumption \\
\hline & Probability HSIL progresses to $\mathrm{CC}$ & 0.33533 & Uniform & $(\text { Rositch, et al })^{3}$ \\
\hline & Probability HSIL regresses to LSIL & 0.01990 & Uniform & $(\text { Cantor, et al) })^{6}$ \\
\hline & Probability HSIL regresses to WELL & 0.08758 & Uniform & $\left(\right.$ Melnikow, et al) ${ }^{21}$ \\
\hline & Probability HSIL stays & 0.55720 & Uniform & Assumption \\
\hline & Probability HSIL progresses to $\mathrm{S}+\mathrm{T}$ & 0.00000 & Uniform & Assumption \\
\hline & Probability S+T progresses to WELL & 1.00000 & Uniform & Assumption \\
\hline & Probability CC progresses to DEATH & 0.01600 & Uniform & $\left(\right.$ Howlader, et al) ${ }^{22}$ \\
\hline & Probability IC stays & 0.98400 & Uniform & Assumption \\
\hline & Probability DEATH stays & 1.00000 & Uniform & Assumption \\
\hline \multirow[t]{18}{*}{ Screening cohort } & Probability WELL progresses to ASCUS & 0.02070 & Uniform & $(\text { Edelman, et al })^{20}$ \\
\hline & Probability WELL stays & 0.97930 & Uniform & Assumption \\
\hline & Probability Well progresses to LSIL & 0.01220 & Uniform & $(\text { Edelman, et al })^{20}$ \\
\hline & Probability ASCUS progresses to HSIL & 0.00450 & Uniform & $(\text { Walker, et al })^{18}$ \\
\hline & Probability ASCUS regresses to WELL & 0.17048 & Uniform & $(\text { Melnikow, et al) })^{21}$ \\
\hline & Probability ASCUS stays & 0.82503 & Uniform & Assumption \\
\hline & Probability LSIL progresses to HSIL & 0.02573 & Uniform & $(\text { Cantor, et al })^{6}$ \\
\hline & Probability LSIL regresses to WELL & 0.07400 & Uniform & $(\text { Cantor, et al })^{6}$ \\
\hline & Probability LSIL stays & 0.90027 & Uniform & Assumption \\
\hline & Probability HSIL progresses to CC & 0.01053 & Uniform & $(\text { Rositch, et al })^{3}$ \\
\hline & Probability HSIL regresses to LSIL & 0.01990 & Uniform & $(\text { Cantor, et al })^{6}$ \\
\hline & Probability HSIL regresses to WELL & 0.08758 & Uniform & $\left(\right.$ Melnikow, et al) ${ }^{21}$ \\
\hline & Probability HSIL stays & 0.00000 & Uniform & Assumption \\
\hline & Probability HSIL progresses to $\mathrm{S}+\mathrm{T}$ & 0.88200 & Uniform & Assumption \\
\hline & Probability $\mathrm{S}+\mathrm{T}$ progresses to WELL & 1.00000 & Uniform & Assumption \\
\hline & Probability $\mathrm{CC}$ progresses to DEATH & 0.01600 & Uniform & $(\text { Howlader, et al })^{22}$ \\
\hline & Probability IC stays & 0.98400 & Uniform & Assumption \\
\hline & Probability DEATH stays & 1.00000 & Uniform & Assumption \\
\hline \multirow[t]{5}{*}{ Costs (All) } & Cost Well & 0.00000 & Fixed & Assumption \\
\hline & Cost No Screening (ASCUS, LSIL, HSIL) & 0.00000 & Fixed & Assumption \\
\hline & Cost to screen & 122.60 & Gamma & $(\text { Medicare })^{23}$ \\
\hline & Cost colposcopy and treatment & 11928.00 & Gamma & $(\text { Medicare })^{23}$ \\
\hline & Cost cervical cancer treatment & 25200.00 & Gamma & $(\text { Subramanian, et al })^{24}$ \\
\hline \multirow[t]{4}{*}{ Preferences (All) } & Well/Undiagnosed LSIL/ASCUS/HSIL & 0.88000 & Triangular & $(\mathrm{Kind}, \mathrm{et} \text { al })^{11}$ \\
\hline & Diagnosed ASCUS/LSIL & 0.85000 & Triangular & $(\text { Zhang, et al) })^{25}$ \\
\hline & Diagnosed HSIL & 0.85000 & Triangular & $(\text { Zhang, et al) })^{25}$ \\
\hline & Screening + Treatment & 0.73000 & Triangular & $\left(\right.$ Zhang, et al) ${ }^{25}$ \\
\hline
\end{tabular}


Multiple iterations are simulated of the cohort progressing through the model, along with simultaneously altered probabilities, preferences, and costs. The cloud is then utilized to produce a cost effectiveness curve, which graphically indicates the likelihood that the result is cost effective, in relation to societal willingness to pay at a certain dollar value. Finally, the net monetary benefit chart is created by converting the realized health benefits by the cost, then with the cost benefit subtracted, which displays, along with a confidence interval, the benefit that can be realized at dollar values of willingness to pay.

\section{RESULTS}

The economic, base case analysis (Table 2) determined that screening in the study population dominates the alternative of no screening, demonstrating both lower average cost, and higher average quality of life.

The per person, calculated difference for screening over none, shows to be a 0.3 QALY improvement, as well as a $\$ 5,402$ reduction in costs, after accounting for time discount.

Table 2: Base case analysis.

\begin{tabular}{|c|c|c|c|c|c|c|}
\hline & \multicolumn{3}{|c|}{ Not discounted } & \multicolumn{3}{|l|}{ Discounted } \\
\hline & Screening & None & Difference & Screening & None & Difference \\
\hline Cost & 4853.9 & 13929.2 & -9075.3 & 3595.8 & 8997.8 & -5402.0 \\
\hline QALYs & 28.0 & 27.6 & 0.4 & 21.1 & 20.8 & 0.3 \\
\hline ICER & & & $(21050)$ & & & (19 578) \\
\hline
\end{tabular}

\section{Sensitivity analysis}

Univariate (Table 3) and multivariate sensitivity analyses were carried out on the model and its parameters. The model demonstrated significant robustness of the base case point estimate ICER, as both the univariate tornado diagram (Figure 2), and probabilistic sensitivity analyses showed a consistent maintenance of both the cost benefits and health benefits of the screening intervention.

Table 3. Univariate sensitivity analysis.

\begin{tabular}{|llll|}
\hline Parameter & ICER & & Difference in ICER \\
\hline Well/Undiagnosed LSIL/ASCUS/HSIL (Preference Weight) & Low Value & High Value & 10805 \\
\hline Cervical Cancer (Preference Weight) & -27686 & -16881 & 105 \\
\hline Probability HSIL progresses to CC (Non-Screening arm) & -13660 & -20839 & 2379 \\
\hline Probability HSIL progresses to S+T (Screening arm) & -19579 & -21476 & 7804 \\
\hline Cervical Cancer Cost & -3524 & -19576 & 3 \\
\hline Screening Cost & -24916 & -8900 & 48161 \\
\hline Colposcopy and Treatment Cost & -20504 & -17725 & 2779 \\
\hline
\end{tabular}

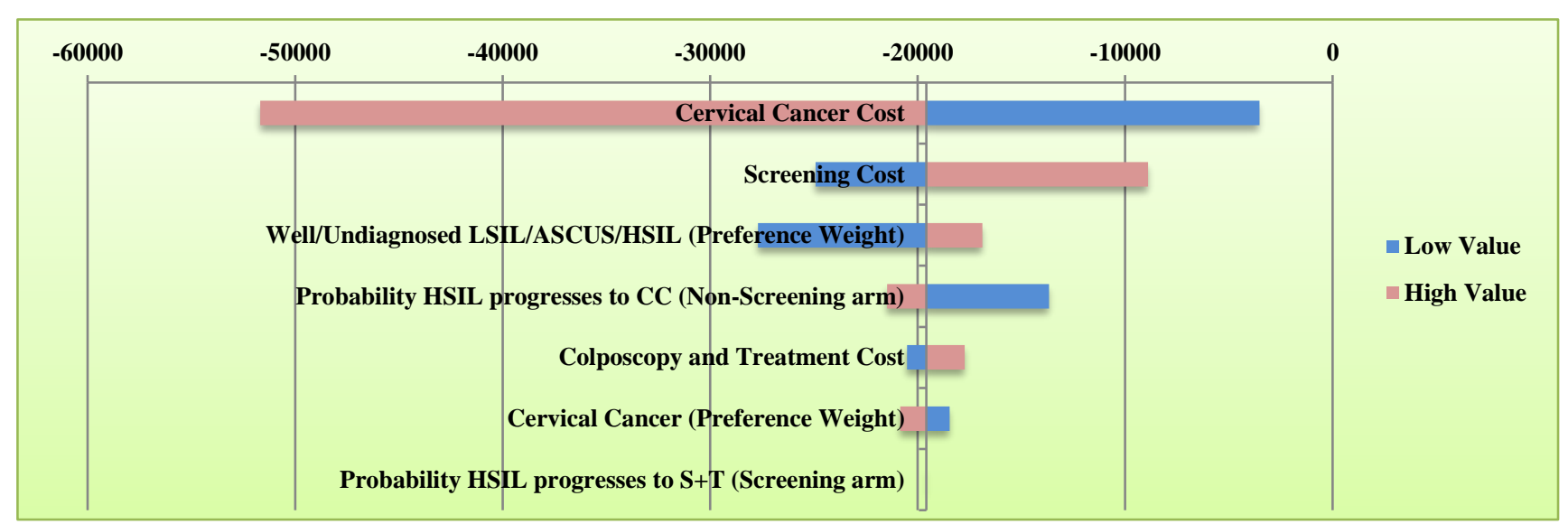

Figure 2. Tornado diagram of univariate analysis. 
Critical selected parameters for the univariate analysis indicated the greatest sensitivity of the model to the costs of cervical cancer and of screening. However, even with plausible variation of costs, with an extended upper limit, the intervention remained cost effective.

This is visible on the tornado diagram, where ICER variation due to parameter variation is illustrated around the study point estimate. Multivariate analysis involved a probabilistic sensitivity analysis that allowed concurrent variation of parameters across the model. A thousand simulated iterations, utilizing the estimated variation of each parameter, of the model produced a cost effectiveness cloud (Figure 3) with $87.1 \%$ of estimated ICER's falling within the fourth quadrant. The cost effectiveness curve (Figure 4) demonstrated a likelihood of cost effectiveness of 0.81 even at a willingness to pay of 0 dollars, with a 0.91 likelihood from $\$ 10,000$ onwards.

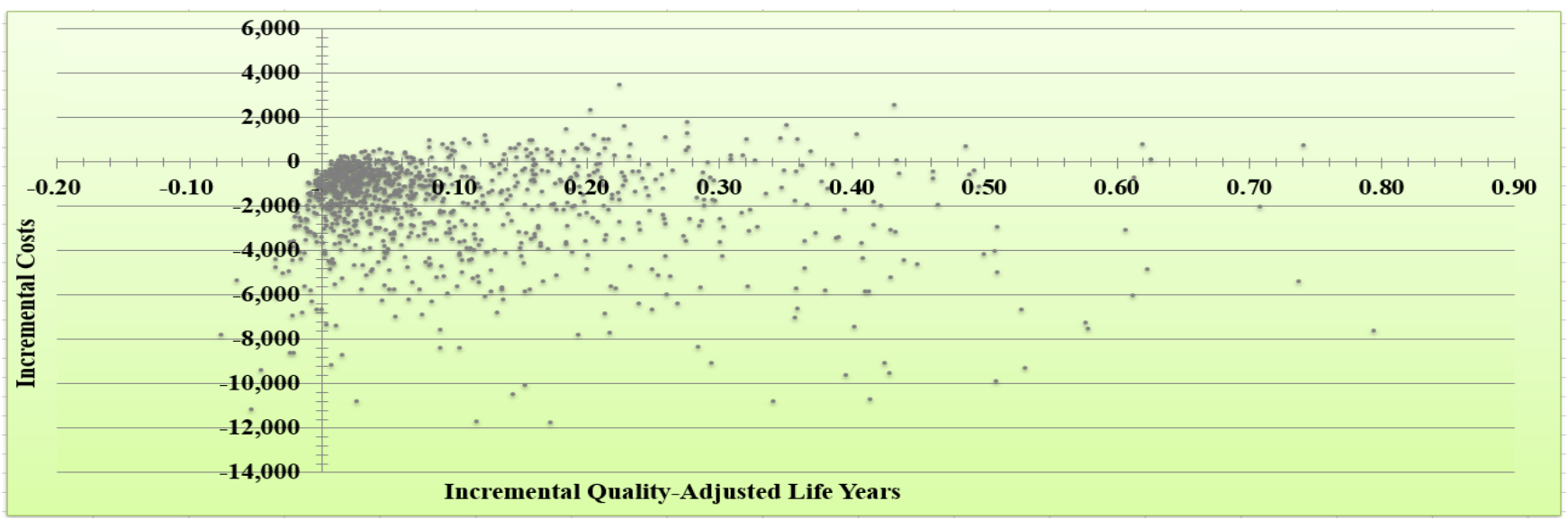

Figure 3: Scatterplot of probabilistic sensitivity analysis simulation.

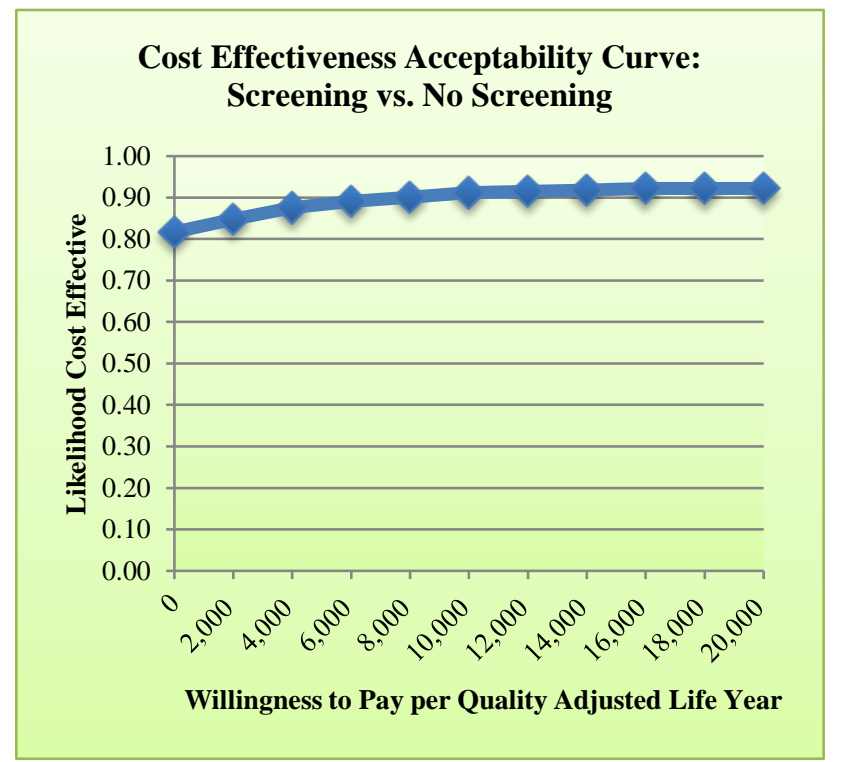

Figure 4. Cost effectiveness acceptability curve from probabilistic sensitivity analysis.

Lastly, the net monetary benefit (Figure 5) shows an NMB of $\$ 2000$ - $\$ 3000$ across a willingness to pay of 0 to 20,000 dollars. The $68 \%$ confidence interval (one standard deviation above and below the estimated line of NMB) remains positive above a $\$ 2000$ willingness to pay.

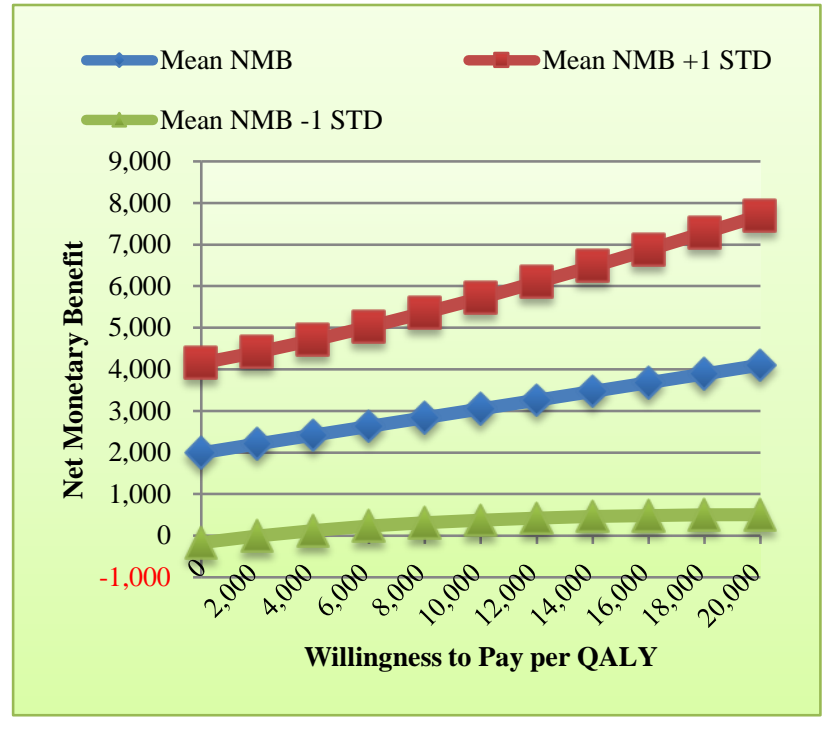

Figure 5. Net monetary benefit as a function of willingness to pay per QALY-screening relative to no screening.

\section{DISCUSSION}

\section{Findings}

The conducted cost effectiveness analysis determined that continued screening for cervical cancer in elderly women 
up to the age of 70 is cost effective, with both an improvement in costs and in health outcomes, therefore dominating the current practice of no screening. This finding is supported by demonstrated robustness through both univariate and multivariate sensitivity analysis, indicating that cost effectiveness holds true over a range of possible costs, disease and treatment probabilities and societal preferences for included health states.

\section{Strengths and limitations}

The study provides a straightforward, and concise analysis of screening effects on cervical cancer impacts through its practical disease progression and treatment Markov model. The model only takes into account states that can be accounted for with probabilities in literature reporting from clinical observations. Thus, the effect of lengthened screening guidelines, as well as the effect of the sensitivity analyses, are understandably plausible.

The main strength of the study lies in its use of the most current epidemiological data as reported by Rositch. ${ }^{3}$ Cost effectiveness can only be as precise and applicable as the data that informs its analysis and conclusions, and for this study, Rositch's revealing findings on the incidence of cervical cancer in the elderly female population in the United States are key to determining the cost effectiveness of screening in the same population.

The short time span of the study allowed the investigator to omit a model stage accounting for deaths from other causes (besides cervical cancer). However, considering the elderly age of the population in question, there remains a likelihood that death from other causes could have an effect upon the outcomes of the analysis run through said model. This should be considered when applying the findings to clinical practice, although the simplicity of the model does help insure its internal validity.

Other concerns, as in many other cost effectiveness studies utilizing secondary data, is the consistency of input costs, probabilities and preference weights. Costs, fortunately, are all sourced from the American Medical Association, are updated yearly, and are intended to represent the entirety of payer cost. Consistent probabilities inputs are much more challenging to find, and unfortunately are derived from multiple sources, and synthesized in order to be representative of the same time span across the model. Likewise, with preference weights, which come from studies on different populations. These are debatable, but unavoidable weaknesses, that the study team has sought to mitigate through considerable discretion on their selection and use.

\section{Policy implications}

The most immediate concern regarding the findings is to inform the stated medical practice guidelines currently being utilized in the US today. Given the startling difference in Dr. Rositch, and team's findings regarding the incidence of cervical malignancy given corrections for hysterectomies in the same population, it is not unexpected to find that screening, up until the age of 70 , is forecast to produce such a dramatic impact. Consequently, this study's cost effectiveness data requires reconciliation with policy maker considerations, to potentially effect a change on the current screening only until age 65 guidelines. ${ }^{1,2}$ Furthermore, findings of the sensitivity analysis provide strong support for the likelihood of a positive impact of improved screening, even if moderate changes to costs or preferences in regard to cervical cancer and medical care occur. This can be regarded as a continuation of the effort of implementing prevention as intervention in the modern medical practice.

\section{ACKNOWLEDGMENTS}

Authors would gratefully acknowledge the support of the doctoral supervisor at the University of Alberta, Dr. Stephanie Montesanti.

Funding: No funding sources

Conflict of interest: None declared

Ethical approval: The study was approved by the Institutional Ethics Committee

\section{REFERENCES}

1. Saslow D, Solomon D, Lawson HW, Killackey M, Kulasingam SL, Cain J, et al. American cancer society, american society for colposcopy and cervical pathology, and american society for clinical pathology screening guidelines for the prevention and early detection of cervical cancer. CA: Cancer J Clinic. 2011;62(3):147-72.

2. USPTF. U.S. Preventive Services Task Force Recommends Screening Most Adult Women for Cervical Cancer. 2017. Available at https://www.uspreventiveservicestaskforce.org/Hom e/GetFile/6/250/cervical-cancer-bulletin/pdf.

3. Rositch AF, Nowak RG, Gravitt PE. Increased age and race-specific incidence of cervical cancer after correction for hysterectomy prevalence in the united states from 2000 to 2009. Cancer.2014;120(13): 2032-8.

4. Apgar BS, Zoschnick L, Wright TC Jr. The 2001 bethesda system terminology. Am Family Physician. 2003;68(10):1992-8.

5. Schlecht NF, Platt RW, Duarte-Franco E, Costa MC, Sobrinho JP, Prado JC, et al. Human papillomavirus infection and time to progression and regression of cervical intraepithelial neoplasia. J National Cancer Inst. 2003;95(17):1336-43.

6. Cantor SB, Atkinson EN, Cardenas-Turanzas M, Benedet JL, Follen M, MacAulay C. Natural history of cervical intraepithelial neoplasia. Acta Cytologica. 2005;49(4):405-5 
7. Benard V. Vital signs: Cervical cancer incidence, mortality, and screening - united states 2007 - 2012. CDC Morbidity and Mortality Weekly Rep. 2014;63(44):1004.

8. Bidus MA, Maxwell GL, Kulasingam S, Rose GS, Elkas JC, Chernofsky M, et al. Cost-effectiveness analysis of liquid-based cytology and human papillomavirus testing in cervical cancer screening. Obstet Gynecol. 2006;107(5):997-1005.

9. Caprara L, Monari F, De Bianchi PS, Amadori A, Bondi A. ASCUS in screening. [ASCUS in screening] Pathol. 2001;93(6):645-50.

10. Esselen KM, Feldman S. Cost-effectiveness of cervical cancer prevention. Clinical Obstet Gynecol. 2013;56(1),55-64.

11. Kind P, Hardman G, Macran S. UK population norms for EQ-5D No. 172. 1993. University of York: Centre for Health Economics.

12. Kulasingam SL, Hughes JP, Kiviat NB, et al. Evaluation of human papillomavirus testing in primary screening for cervical abnormalities: Comparison of sensitivity, specificity, and frequency of referral. JAMA. 2002;288(14):1749-57.

13. Myers ER, McCrory DC, Nanda K, Bastian L, Matchar DB. Mathematical model for the natural history of human papillomavirus infection and cervical carcinogenesis. Am J Epidemiol. 2000;51(12),1158-71.

14. Plummer M, Schiffman M, Castle PE, MaucortBoulch D, Wheeler CM, ALTS Group. A 2-year prospective study of human papillomavirus persistence among women with a cytological diagnosis of atypical squamous cells of undetermined significance or low-grade squamous intraepithelial lesion. J Infect Disease. 2007;195(11):1582-9.

15. Taylor DC, Pawar V, Kruzikas D, Gilmore KE, Pandya A, Iskandar R, et al. Methods of model calibration: Observations from a mathematical model of cervical cancer. Pharmacoeco. 2010;28(11):9951000.

16. Vijayaraghavan A, Efrusy MB, Goodman KA, Santas CC, Huh WK. Cost-effectiveness of using human papillomavirus $16 / 18$ genotype triage in cervical cancer screening. Gynecol Oncol. 2010;119(2):237-42.

17. Vink MA, Bogaards JA, van Kemenade FJ, de Melker HE, Meijer CJ, Berkhof J. Clinical progression of high-grade cervical intraepithelial neoplasia: Estimating the time to preclinical cervical cancer from doubly censored national registry data. Am J Epidemiol. 2013;178(7), 1161-9.
18. Walker JL, Wang SS, Schiffman M, Solomon D. Predicting absolute risk of CIN3 during postcolposcopic follow-up: Results from the ASCUSLSIL triage study (ALTS). Am J Obstet Gynecol. 2006;195(2):341-8.

19. Zhao Z, Pan X, Lv S, Xie Y, Zhang S, Qiao Y, et al. Quality of life in women with cervical precursor lesions and cancer: A prospective, 6-month, hospitalbased study in china. Chinese $\mathbf{J}$ Cancer. 2013;33(7):339-45.

20. Edelman M, Fox AS, Alderman EM, Neal W, Shapiro A, Silver EJ, et al. Cervical Papanicolaou smear abnormalities in inner city Bronx adolescents: prevalence, progression, and immune modifiers. Cancer. 1999;87(4):184-9.

21. Melnikow J, Nuovo J, Willan AR, Chan BK, Howell LP. Natural history of cervical squamous intraepithelial lesions: a meta-analysis. Obstet Gynecol. 1998:92(4 Pt 2):727-35.

22. Howlader N, Noone AM, Krapcho M, Garshell J, Miller D, Altekruse SF, et al, eds. SEER Cancer Statistics Review, 1975-2011, National Cancer Institute. Bethesda, MD, https://seer.cancer.gov/csr/1975_2011/, based on November 2013 SEER data submission, posted to the SEER web site, April 2014.

23. Centers for Medicare and Medicaid Service. Physician Fee Schedule Look-Up Tool. 2015. Accessed February 25, 2015. Available at: https://www.cms.gov/Medicare/Medicare-Fee-forService-Payment/PFSlookup/.

24. Subramanian S, Trogdon J, Ekwueme DU, Gardner JG, Whitmire JT, Rao C. Cost of cervical cancer treatment: implications for providing coverage to low-income women under the Medicaid expansion for cancer care. Womens Health Issues. 2010;20(6):400-5.

25. Zhang $\mathrm{X}$, Zhang L, Tian $\mathrm{C}$, Yang L, Wang Z. Genetic variants and risk of cervical cancer: epidemiological evidence, meta-analysis and research review. BJOG: Int J Obstet Gynaecol. 2014;121(6):664-73.

Cite this article as: Quon JC. Cost effectiveness analysis of cervical cancer screening in women until age 70. Int J Reprod Contracept Obstet Gynecol 2018;7:3899-905. 